\title{
Efficient energy stable numerical schemes for a phase field moving contact line model
}

\author{
Jie Shen ${ }^{\mathrm{a}}$, Xiaofeng Yang ${ }^{\mathrm{b}}$, Haijun $\mathrm{Yu}^{\mathrm{c}, *}$ \\ ${ }^{a}$ Department of Mathematics, Purdue University, West Lafayette, IN 47906-1957 \\ ${ }^{b}$ Department of Mathematics, University of South Carolina, Columbia SC 29208 \\ ${ }^{c}$ LSEC \& ICMSEC, Academy of Mathematics and Systems Science, Beijing 100190, China
}

\begin{abstract}
In this paper, we present two efficient energy stable schemes to solve a phase field model incorporating moving contact line. The model is a coupled system that consists of incompressible Navier-Stokes equations with a generalized Navier boundary condition and CahnHilliard equation in conserved form. In both schemes the projection method is used to deal with the Navier-Stokes equations and stabilization approach is used for the non-convex Ginzburg-Landau bulk potential. By some subtle explicit-implicit treatments, we obtain a linear coupled energy stable scheme for systems with dynamic contact line conditions and a linear decoupled energy stable scheme for systems with static contact line conditions. An efficient spectral-Galerkin spatial discretization method is implemented to verify the accuracy and efficiency of proposed schemes. Numerical results show that the proposed schemes are very efficient and accurate.
\end{abstract}

Keywords: moving contact line, phase field, Navier-Stokes equations, Cahn-Hilliard equation, splitting methods

\section{Introduction}

Phase-field/diffuse-interface models, whose origin can be traced back to Rayleigh (1892) and Waals (1893), have become one of the major tools to deal with many dynamical processes in material/biological morphology, in particular, the multiphase fluid systems that we are interested in this paper. The typical phase field model can be described by either the Allen-Cahn equation (see Bray (1994)) or the Cahn-Hilliard equation (Cahn and Hilliard, 1958) based on energetic variational approaches. The Allen-Cahn equation is a second-order equation, which is easier to solve numerically but does not conserve the volume fraction, while the Cahn-Hillard equation is a fourth-order equation which conserves the volume

\footnotetext{
*Corresponding author.

Email addresses: shen@math.purdue.edu (Jie Shen), xfyang@math.sc.edu (Xiaofeng Yang), hyu@lsec.cc.ac.cn (Haijun Yu)

Preprint submitted to Journal of Computational Physics

December 31, 2014
}

(C) 2015. This manuscript version is made available under the Elsevier user license http:/www.elsevier.com/open-access/userlicense/1.0/ 
fraction but is relatively harder to solve numerically. A particular advantage of the phasefield approach is that the derived models are usually well-posed nonlinear partial differential equations that satisfy thermodynamics-consistent energy dissipation laws, which makes it possible to carry out mathematical analysis and further design numerical schemes which satisfy corresponding discrete energy dissipation laws. Thus phase field models have been the subject of many theoretical and numerical investigations recently (cf., for instance, Du and Nicolaides (1991), Eyre (1998), Furihata (2001), Liu and Shen (2003), Kessler et al. (2004), He et al. (2007), Shen and Yang (2010b,c), Yue et al. (2010), Condette et al. (2011), Shen (2011), Gao and Wang (2012), Salgado (2013), Shen and Yang (2014a,b)).

When the fluid-fluid interface touches a solid wall, it creates a moving contact line (MCL) problem that exists in many physical processes, for instance, wetting, coating, painting, etc. In this situation, it is well-known that the no-slip boundary condition for the NavierStokes equations is no longer applicable, otherwise, a non-physical velocity discontinuity will occur at the MCL (see e.g. Moffatt (1964), Dussan V. and Davis (1974), Dussan (1979)). Simulations by Koplik et al. (1988, 1989), Thompson and Robbins (1989), among others, using Molecular Dynamics (MD) showed that nearly complete slip happens near the MCL. To investigate the complex behavior at MCLs, microscopic-macroscopic hybrid simulations were carried out by Hadjiconstantinou (1999), Ren and E (2005), etc. This approach is powerful but computationally expensive for macroscopic applications. On the other hand, a set of accurate boundary conditions for the MCL problem in the context of phase field model was derived by Qian et al. (2003), where they proposed a general Navier boundary condition (GNBC) and a dynamic contact line condition for a macroscopic model consisting of the Navier-Stokes equations and the Cahn-Hilliard equation. An explicit numerical scheme, which dictates a very small time step, was used to solve the Cahn-Hilliard equation to compare the results with MD simulations.

Recently, several attempts were made to improve the numerical stability and efficiency of solving the Navier-Stokes and Cahn-Hilliard coupled system for MCL problems. In He et al. (2011), the authors proposed an operator splitting method with a least-squares finite element method for one of the sub-steps. The authors of Dong (2012) and Dong and Shen (2012) constructed some decoupled schemes for systems with variable density, however they did not provide any theoretical proof of discrete energy law for the decoupled schemes with dynamic contact line conditions. In Gao and Wang (2012, 2014), Salgado (2013) and Aland and Feng (2014), the authors developed some energy stable schemes for the moving contact line problem with constant and/or variable densities. However, their schemes require solving a coupled nonlinear system for the phase function and velocity.

In this paper, we consider a phase-field moving contact line model which is a conserved version of the model proposed by Qian et al. (2003, 2006). We construct two energy stable temporal schemes for this model. One is a linear coupled scheme for systems with dynamic contact line conditions, the other is a linear decoupled scheme for systems with static contact line conditions. We then implement a Fourier-Legendre Galerkin approximation to investigate the efficiency and accuracy for the two schemes. 


\section{A Navier-Stokes Cahn-Hilliard coupled model in conserved form}

We consider the moving contact line dynamics of a two-phase incompressible, immiscible fluid in a physical domain denoted by $\Omega$ with boundary $\Gamma$. It is showed in Qian et al. (2003, 2006) that this problem can be modeled by a Navier-Stokes Cahn-Hilliard coupled system (NSCH) with a general Navier boundary condition. A non-dimensional, conserved version of the system is given below.

Incompressible Navier-Stokes equations for hydrodynamics:

$$
\begin{gathered}
R\left(\boldsymbol{u}_{t}+\boldsymbol{u} \cdot \nabla \boldsymbol{u}\right)=\Delta \boldsymbol{u}-\nabla p-B \phi \nabla \mu, \\
\nabla \cdot \boldsymbol{u}=0, \\
\boldsymbol{u} \cdot \boldsymbol{n}=0 \quad \text { on } \Gamma, \\
l(\phi)\left(\boldsymbol{u}_{\tau}-\boldsymbol{u}_{w}\right)+\partial_{n} \boldsymbol{u}_{\tau}-B L(\phi) \nabla_{\tau} \phi=0 \quad \text { on } \Gamma .
\end{gathered}
$$

Cahn-Hilliard equation for the dynamics of phase variable:

$$
\begin{gathered}
\phi_{t}+\nabla \cdot(\boldsymbol{u} \phi)=M \Delta \mu, \\
\mu=-\varepsilon \Delta \phi+f(\phi), \\
\partial_{n} \mu=0 \text { on } \Gamma, \\
\phi_{t}+\boldsymbol{u}_{\tau} \cdot \nabla_{\tau} \phi=-\gamma L(\phi) \text { on } \Gamma .
\end{gathered}
$$

In the above system, the unknowns are: $\boldsymbol{u}$ - the fluid velocity, $p$ - the pressure, $\phi$ the phase-field variable, $\mu$ - the chemical potential. The function $L(\phi)$ in equation $(2.8)$ is given by

$$
L(\phi)=\varepsilon \partial_{n} \phi+g^{\prime}(\phi),
$$

where $g(\phi)$ is the boundary interfacial energy; $l(\phi) \geq 0$ is a given coefficient function; the function $f(\phi)=F^{\prime}(\phi)$, with $F(\phi)$ being the Ginzburg-Landau bulk potential. More precisely, $F$ and $g$ are defined as

$$
F(\phi)=\frac{1}{4 \varepsilon}\left(\phi^{2}-1\right)^{2}, \quad g(\phi)=-\frac{\sqrt{2}}{3} \cos \theta_{s} \sin \left(\frac{\pi}{2} \phi\right),
$$

where $\theta_{s}$ is the static contact angle. In equations (2.1)-(2.9), bold face letters denote vector variables, $\nabla$ denotes the gradient operator, $\boldsymbol{n}$ is the outward normal direction on boundary $\Gamma$, scalar operator $\partial_{n}=\boldsymbol{n} \cdot \nabla$ is the partial derivative along direction $\boldsymbol{n}, \tau$ is the boundary tangential direction, and vector operator $\nabla_{\tau}=\nabla-(\boldsymbol{n} \cdot \nabla) \boldsymbol{n}$ is the gradient along tangential direction, $\boldsymbol{u}_{w}$ is the boundary wall velocity, $\boldsymbol{u}_{\tau}$ is the boundary fluid velocity in tangential direction. From (2.3), we have $\boldsymbol{u}=\boldsymbol{u}_{\tau}$ on boundary $\Gamma$.

There are six non-dimensional parameters in this system. $R$ is the Reynolds number, $B$ denotes the strength of the capillary force comparing to the Newtonian fluid stress, $M$ is the mobility coefficient, $\gamma$ is a boundary relaxation coefficient, $l(\phi)$ is the ratio of domain size to boundary slip length, $\varepsilon$ is the ratio between interface thickness and domain size. Similar 
parameters are used by He et al. (2011) and Gao and Wang (2012), with $M, \gamma$ and $l(\phi)$ here denoted by $\mathcal{L}_{d}, \mathcal{V}_{s}$ and $\mathcal{L}_{s}(\phi)^{-1}$ in He et al. (2011), correspondingly.

The GNBC (2.4) and the dynamic contact line condition (2.8) were introduced in Qian et al. (2003). When $\gamma \rightarrow+\infty$, the dynamic contact line condition (2.8) is reduced to the static contact line condition

$$
L(\phi)=0 \quad \text { on } \Gamma,
$$

and the GNBC is reduced to the Navier boundary condition (NBC)

$$
l(\phi)\left(\boldsymbol{u}_{\tau}-\boldsymbol{u}_{w}\right)+\partial_{n} \boldsymbol{u}_{\tau}=0 \quad \text { on } \Gamma .
$$

If one further sets $g^{\prime}(\phi) \equiv 0$, the system is reduced to a phase-field model without contact line effects. If we take $l(\phi) \rightarrow+\infty$ in equation (2.12), then the NBC is reduced to the traditional no-slip boundary condition.

Note that, in equation (2.5), a conserved form $\nabla \cdot(\boldsymbol{u} \phi)$ for the phase variable $\phi$ is used. Correspondingly, we use capillary force $-B \phi \nabla \mu$ in (2.1), which is different from $B \mu \nabla \phi$ used in Qian et al. $(2003,2006)$. The difference $-B \nabla(\phi \mu)$ is absorbed into pressure $p$. By using this formulation, it is more convenient to establish the following energy dissipation law, and to design energy stable numerical schemes.

Here and after, for any function $f, g \in H^{1}(\Omega)$, we use $(f, g)$ to denote $\int_{\Omega} f g \mathrm{~d} x,(f, g)_{\Gamma}$ to denote $\int_{\Gamma} f g \mathrm{~d} x$, and let $\|f\|^{2}=(f, f),\|f\|_{\Gamma}^{2}=(f, f)_{\Gamma}$.

Theorem 1. The NSCH system (2.1)-(2.8) is a dissipative system satisfying the following energy dissipation law

$$
\frac{\mathrm{d}}{\mathrm{d} t} E_{t o t}=-\|\nabla \boldsymbol{u}\|^{2}-M B\|\nabla \mu\|^{2}-B \gamma\|L(\phi)\|_{\Gamma}^{2}-\left\|l(\phi)^{1 / 2} \boldsymbol{u}_{s}\right\|_{\Gamma}^{2}-\left(l(\phi) \boldsymbol{u}_{s}, \boldsymbol{u}_{w}\right)_{\Gamma},
$$

where $\boldsymbol{u}_{s}=\boldsymbol{u}-\boldsymbol{u}_{w}$ is the velocity slip on boundary $\Gamma, E_{t o t}=E_{k}[\boldsymbol{u}]+E_{b}[\phi]+E_{s}[\phi]$, and

$$
E_{k}[\boldsymbol{u}]=\frac{R}{2}\|\boldsymbol{u}\|^{2}, \quad E_{b}[\phi]=\frac{B \varepsilon}{2}\|\nabla \phi\|^{2}+B(F(\phi), 1), \quad E_{s}[\phi]=B(g(\phi), 1)_{\Gamma} .
$$

Remark 1. The terms except the last one on the right hand side of (2.13) are diffusion or relaxation terms. The last term is a boundary interaction term, its sign is not definite. The energy $E_{s}[\phi]$ defined in equation (2.14) is not positive definite. However, one can make it positive definite by adding a positive constant into function $g$, since only the derivative of $g$ is involved in the governing equations.

Proof. By taking the inner product of equation (2.1) with $\boldsymbol{u}$, using the zero flux boundary condition (2.3) and the incompressible condition (2.2), we get

$$
\frac{R}{2} \frac{\mathrm{d}}{\mathrm{d} t}\|\boldsymbol{u}\|^{2}=\left(\partial_{n} \boldsymbol{u}, \boldsymbol{u}\right)_{\Gamma}-\|\nabla \boldsymbol{u}\|^{2}-B(\phi \nabla \mu, \boldsymbol{u}) .
$$

Taking the inner product of equation (2.5) with $B \mu$, and using boundary conditions (2.3) and (2.7), we get

$$
B\left(\phi_{t}, \mu\right)-B(\boldsymbol{u} \phi, \nabla \mu)=-M B\|\nabla \mu\|^{2} .
$$


Taking the inner product of equation (2.6) with $-B \phi_{t}$, we have

$$
-B\left(\mu, \phi_{t}\right)=B \varepsilon\left(\partial_{n} \phi, \phi_{t}\right)_{\Gamma}-\frac{B \varepsilon}{2} \frac{\mathrm{d}}{\mathrm{d} t}\|\nabla \phi\|^{2}-B \frac{\mathrm{d}}{\mathrm{d} t}(F(\phi), 1) .
$$

Summing up equations (2.15)-(2.17), we obtain

$$
\begin{aligned}
\frac{R}{2} \frac{\mathrm{d}}{\mathrm{d} t}\|\boldsymbol{u}\|^{2}+\frac{B \varepsilon}{2} \frac{\mathrm{d}}{\mathrm{d} t}\|\nabla \phi\|^{2}+B \frac{\mathrm{d}}{\mathrm{d} t}(F(\phi), 1)= & -\|\nabla \boldsymbol{u}\|^{2}-M B\|\nabla \mu\|^{2} \\
& +\left(\partial_{n} \boldsymbol{u}, \boldsymbol{u}\right)_{\Gamma}+B \varepsilon\left(\partial_{n} \phi, \phi_{t}\right)_{\Gamma} .
\end{aligned}
$$

Then, we use boundary condition (2.4) and (2.8)-(2.9) to derive

$$
\begin{aligned}
\left(\partial_{n} \boldsymbol{u}, \boldsymbol{u}\right)_{\Gamma} & =\left(\partial_{n} \boldsymbol{u}_{\tau}, \boldsymbol{u}_{\tau}\right)_{\Gamma}=\left(B L(\phi) \nabla_{\tau} \phi-l(\phi)\left(\boldsymbol{u}_{\tau}-\boldsymbol{u}_{w}\right), \boldsymbol{u}_{\tau}\right)_{\Gamma} \\
& =B\left(L(\phi) \nabla_{\tau} \phi, \boldsymbol{u}_{\tau}\right)_{\Gamma}-\left(l(\phi) \boldsymbol{u}_{s}, \boldsymbol{u}_{s}+\boldsymbol{u}_{w}\right)_{\Gamma}, \\
B \varepsilon\left(\partial_{n} \phi, \phi_{t}\right)_{\Gamma} & =B\left(L(\phi)-g^{\prime}(\phi), \phi_{t}\right)_{\Gamma} \\
& =B\left(L(\phi), \phi_{t}\right)_{\Gamma}-B\left(g^{\prime}(\phi), \phi_{t}\right)_{\Gamma} \\
& =B\left(L(\phi),-\boldsymbol{u}_{\tau} \cdot \nabla_{\tau} \phi-\gamma L(\phi)\right)_{\Gamma}-B \frac{\mathrm{d}}{\mathrm{d} t}(g(\phi), 1)_{\Gamma} \\
& =-B\left(L(\phi) \nabla_{\tau} \phi, \boldsymbol{u}_{\tau}\right)_{\Gamma}-B \gamma\|L(\phi)\|_{\Gamma}^{2}-B \frac{\mathrm{d}}{\mathrm{d} t}(g(\phi), 1)_{\Gamma} .
\end{aligned}
$$

Summing up (2.18), (2.19) and (2.20), we get the desired energy estimate (2.13).

\section{Two energy stable schemes}

\subsection{A linear coupled energy stable scheme}

There are two popular approaches to handle the non-convex Ginzburg-Landau potential $F(\phi)$ in equation (2.10). One is the convex splitting method (cf. Elliott and Stuart (1993) and Eyre (1998)), another is the stabilization method (cf. Zhu et al. (1999); Xu and Tang (2006); Shen and Yang (2010b)). Here we adopt the later one, which does not require solving a nonlinear equation. The unconditional stability of the stabilization method requires that the second derivative of $F(\phi)$ to be bounded. However, this is not satisfied by the GinzburgLaudau potential. Since we are only interested in $\phi \in[-1,1]$, and it is proved by Caffarelli and Muler (1995) that a truncated $F(\phi)$ with quadratic growth at infinity also guarantees the boundless of $\phi$ in Cahn-Hilliard equation. So it is a common practice to modify $F(\phi)$ to have a quadratic growth rate for $|\phi|>1$ (see e.g. Shen and Yang (2010b), Condette et al. (2011)). Without loss of generality, we introduce the following $\hat{F}(\phi)$ to replace $F(\phi)$ :

$$
\hat{F}(\phi)=\frac{1}{4 \varepsilon} \begin{cases}2(\phi+1)^{2}, & \text { if } \phi<-1, \\ \left(\phi^{2}-1\right)^{2}, & \text { if }-1 \leq \phi \leq 1, \\ 2(\phi-1)^{2}, & \text { if } \phi>1\end{cases}
$$


Correspondingly, we define $\hat{f}(\phi)=\hat{F}^{\prime}(\phi)$ and

$$
L_{1}=\max _{\phi \in \mathbb{R}}\left|\hat{f}^{\prime}(\phi)\right|=\frac{2}{\varepsilon}, \quad L_{2}=\max _{\phi \in \mathbb{R}}\left|g^{\prime \prime}(\phi)\right|=\frac{\sqrt{2} \pi^{2}}{12}\left|\cos \theta_{s}\right| .
$$

Now we are ready to present a linear coupled (LC) scheme for the NSCH system with dynamic contact line conditions. We use $\delta t$ to denote time step size, use a superscript $n$ on $\boldsymbol{u}, \tilde{\boldsymbol{u}}, p, \phi, \mu$ etc to denote approximations of corresponding variables at time $n \delta t$. Given $\boldsymbol{u}^{n}, \phi^{n}, p^{n}$, the LC scheme calculates $\boldsymbol{u}^{n+1}, \phi^{n+1}, p^{n+1}$ and $\mu^{n+1}$ in two steps.

(i) We first solve $\tilde{\boldsymbol{u}}^{n+1}, \phi^{n+1}, \mu^{n+1}$ from

$$
\begin{gathered}
R\left(\frac{\tilde{\boldsymbol{u}}^{n+1}-\boldsymbol{u}^{n}}{\delta t}+\boldsymbol{u}^{n} \cdot \nabla \tilde{\boldsymbol{u}}^{n+1}\right)=\Delta \tilde{\boldsymbol{u}}^{n+1}-\nabla p^{n}-B \phi^{n} \nabla \mu^{n+1}, \\
\mu^{n+1}=-\varepsilon \Delta \phi^{n+1}+\hat{f}\left(\phi^{n}\right)+s_{1}\left(\phi^{n+1}-\phi^{n}\right) \\
\frac{\phi^{n+1}-\phi^{n}}{\delta t}+\nabla \cdot\left(\tilde{\boldsymbol{u}}^{n+1} \phi^{n}\right)=M \Delta \mu^{n+1}
\end{gathered}
$$

with boundary conditions

$$
\begin{gathered}
\tilde{\boldsymbol{u}}^{n+1} \cdot \boldsymbol{n}=0 \quad \text { on } \Gamma, \\
\partial_{n} \tilde{\boldsymbol{u}}_{\tau}^{n+1}+l\left(\phi^{n}\right) \tilde{\boldsymbol{u}}_{s}^{n+1}-B \tilde{L}^{n+1} \nabla_{\tau} \phi^{n}=0 \quad \text { on } \Gamma, \\
\partial_{n} \mu^{n+1}=0 \quad \text { on } \Gamma, \\
\frac{\phi^{n+1}-\phi^{n}}{\delta t}+\tilde{\boldsymbol{u}}_{\tau}^{n+1} \cdot \nabla_{\tau} \phi^{n}=-\gamma \tilde{L}^{n+1} \text { on } \Gamma,
\end{gathered}
$$

where

$$
\tilde{L}^{n+1}=\varepsilon \partial_{n} \phi^{n+1}+g^{\prime}\left(\phi^{n}\right)+s_{2}\left(\phi^{n+1}-\phi^{n}\right),
$$

and $s_{1}, s_{2}$ are two positive stabilizing coefficients.

(ii) In the second step, we update $\boldsymbol{u}^{n+1}, p^{n+1}$ from

$$
\begin{gathered}
R \frac{\boldsymbol{u}^{n+1}-\tilde{\boldsymbol{u}}^{n+1}}{\delta t}+\nabla\left(p^{n+1}-p^{n}\right)=0, \\
\nabla \cdot \boldsymbol{u}^{n+1}=0, \\
\boldsymbol{u}^{n+1} \cdot \boldsymbol{n}=0 \quad \text { on } \Gamma .
\end{gathered}
$$

Some remarks are in order.

(i) A pressure-correction scheme (cf., for instance, Guermond et al. (2006)) is used to decouple the computation of the pressure from that of the velocity. 
(ii) We recall that $f(\phi)=\frac{1}{\varepsilon} \phi\left(\phi^{2}-1\right)$, so the explicit treatment of this term usually leads to a severe restriction on the time step $\delta t$ when $\varepsilon \ll 1$. Thus we introduce in (3.4) a "stabilizing" term to improve the stability while preserving the simplicity. It allows us to treat the nonlinear term explicitly without suffering from any time step constraint (Shen and Yang, 2010a,b,c). Note that this stabilizing term introduces an extra consistent error of order $O(\delta t)$ in a small region near the interface, but this error is of the same order as the error introduced by treating $f(\phi)$ explicitly, so the overall truncation error is essentially of the same order with or without the stabilizing term. A similar approach is applied in the contact line boundary condition (3.9), (3.10). These treatments allow us to prove a discrete energy dissipation law.

Theorem 2. Assume $\boldsymbol{u}_{w}=0, s_{1} \geq L_{1} / 2$ and $s_{2} \geq L_{2} / 2$. Then, the scheme (3.3)-(3.13) is energy stable in the sense that

$$
E_{t o t}^{n+1}-E_{t o t}^{n} \leq 0, \quad n=0,1,2, \ldots,
$$

where

$$
E_{\text {tot }}^{n}=E_{k}\left[\boldsymbol{u}^{n}\right]+\hat{E}_{b}\left[\phi^{n}\right]+E_{s}\left[\phi^{n}\right]+\frac{\delta t^{2}}{2 R}\left\|\nabla p^{n}\right\|^{2}, \quad \hat{E}_{b}[\phi]=\frac{B \varepsilon}{2}\|\nabla \phi\|^{2}+B(\hat{F}(\phi), 1) .
$$

Proof. (i) Taking inner product of (3.3) with $\tilde{\boldsymbol{u}}^{n+1}$, using identities

$$
\begin{gathered}
(a-b, 2 a)=|a|^{2}-|b|^{2}+|a-b|^{2}, \\
(\boldsymbol{u} \cdot \nabla \boldsymbol{v}, \boldsymbol{v})=0, \quad \forall \boldsymbol{u}, \boldsymbol{v} \in H^{1}(\Omega) \text { and } \boldsymbol{u} \text { satisfies (2.2)-(2.3), }
\end{gathered}
$$

we have

$$
\begin{aligned}
\frac{R}{2 \delta t}\left(\left\|\tilde{\boldsymbol{u}}^{n+1}\right\|^{2}-\left\|\boldsymbol{u}^{n}\right\|^{2}+\left\|\tilde{\boldsymbol{u}}^{n+1}-\boldsymbol{u}^{n}\right\|^{2}\right)=- & \left\|\nabla \tilde{\boldsymbol{u}}^{n+1}\right\|^{2}+\left(\partial_{n} \tilde{\boldsymbol{u}}^{n+1}, \tilde{\boldsymbol{u}}^{n+1}\right)_{\Gamma} \\
& -\left(\nabla p^{n}, \tilde{\boldsymbol{u}}^{n+1}\right)-B\left(\phi^{n} \nabla \mu^{n+1}, \tilde{\boldsymbol{u}}^{n+1}\right) .
\end{aligned}
$$

(ii) Taking inner product of (3.5) with $B \mu^{n+1}$, and using (3.6), (3.8), we have

$$
B\left(\frac{\phi^{n+1}-\phi^{n}}{\delta t}, \mu^{n+1}\right)-B\left(\tilde{\boldsymbol{u}}^{n+1} \phi^{n}, \nabla \mu^{n+1}\right)=-B M\left\|\nabla \mu^{n+1}\right\|^{2} .
$$

(iii) Taking inner product of (3.4) with $-B\left(\phi^{n+1}-\phi^{n}\right) / \delta t$, we have

$$
\begin{aligned}
-B\left(\mu^{n+1}, \frac{\phi^{n+1}-\phi^{n}}{\delta t}\right)=- & B \frac{\varepsilon}{2 \delta t}\left(\left\|\nabla \phi^{n+1}\right\|^{2}-\|\nabla \phi\|^{2}+\left\|\nabla\left(\phi^{n+1}-\phi^{n}\right)\right\|^{2}\right) \\
+ & B \varepsilon\left(\partial_{n} \phi^{n+1}, \frac{\phi^{n+1}-\phi^{n}}{\delta t}\right)_{\Gamma} \\
& -B\left(\hat{f}\left(\phi^{n}\right)+s_{1}\left(\phi^{n+1}-\phi^{n}\right), \frac{\phi^{n+1}-\phi^{n}}{\delta t}\right) .
\end{aligned}
$$


For the boundary integral terms in (3.18), by using (3.10), we have

$$
\varepsilon\left(\partial_{n} \phi^{n+1}, \frac{\phi^{n+1}-\phi^{n}}{\delta t}\right)_{\Gamma}=\left(\tilde{L}^{n+1}-\left(g^{\prime}\left(\phi^{n}\right)+s_{2}\left(\phi^{n+1}-\phi^{n}\right)\right), \frac{\phi^{n+1}-\phi^{n}}{\delta t}\right)_{\Gamma} .
$$

By Taylor expansions of $\hat{F}(\phi)$ and $g(\phi)$, we know there exist $\xi, \zeta \in[-1,1]$, such that

$$
\begin{aligned}
& \hat{f}\left(\phi^{n}\right)\left(\phi^{n+1}-\phi^{n}\right)=\hat{F}\left(\phi^{n+1}\right)-\hat{F}\left(\phi^{n}\right)-\frac{\hat{f}^{\prime}(\xi)}{2}\left(\phi^{n+1}-\phi^{n}\right)^{2}, \\
& g^{\prime}\left(\phi^{n}\right)\left(\phi^{n+1}-\phi^{n}\right)=g\left(\phi^{n+1}\right)-g\left(\phi^{n}\right)-\frac{g^{\prime \prime}(\zeta)}{2}\left(\phi^{n+1}-\phi^{n}\right)^{2} .
\end{aligned}
$$

Combining equations (3.18), (3.19), (3.20) and (3.21), we get

$$
\begin{aligned}
-B\left(\mu^{n+1}, \frac{\phi^{n+1}-\phi^{n}}{\delta t}\right)= & -\frac{B \varepsilon}{2 \delta t}\left(\left\|\nabla \phi^{n+1}\right\|^{2}-\|\nabla \phi\|^{2}+\left\|\nabla\left(\phi^{n+1}-\phi^{n}\right)\right\|^{2}\right) \\
& -B\left(\frac{\hat{F}\left(\phi^{n+1}\right)-\hat{F}\left(\phi^{n}\right)}{\delta t}, 1\right)-B\left(s_{1}-\frac{\hat{f}^{\prime}(\xi)}{2}, \frac{\left(\phi^{n+1}-\phi^{n}\right)^{2}}{\delta t}\right) \\
& -B\left(\frac{g\left(\phi^{n+1}\right)-g\left(\phi^{n}\right)}{\delta t}, 1\right)_{\Gamma}-B\left(s_{2}-\frac{g^{\prime \prime}(\zeta)}{2}, \frac{\left(\phi^{n+1}-\phi^{n}\right)^{2}}{\delta t}\right)_{\Gamma} \\
& +B\left(\tilde{L}^{n+1}, \frac{\phi^{n+1}-\phi^{n}}{\delta t}\right)_{\Gamma} \cdot
\end{aligned}
$$

(iv) For the last boundary term in (3.22) and the boundary term in (3.16), using equations (3.9),(3.7) and noticing that $\tilde{\boldsymbol{u}}_{\tau}^{n+1}-\tilde{\boldsymbol{u}}_{w}^{n+1}=\tilde{\boldsymbol{u}}_{s}^{n+1}, \tilde{\boldsymbol{u}}_{w}^{n+1}=\mathbf{0}$, we obtain

$$
\begin{aligned}
B\left(\tilde{L}^{n+1}, \frac{\phi^{n+1}-\phi^{n}}{\delta t}\right)_{\Gamma} & =B\left(\tilde{L}^{n+1},-\gamma \tilde{L}^{n+1}-\tilde{\boldsymbol{u}}_{\tau}^{n+1} \cdot \nabla_{\tau} \phi^{n}\right)_{\Gamma} \\
& =-B \gamma\left\|\tilde{L}^{n+1}\right\|_{\Gamma}^{2}-B\left(\tilde{L}^{n+1} \nabla_{\tau} \phi^{n}, \tilde{\boldsymbol{u}}_{\tau}^{n+1}\right)_{\Gamma},
\end{aligned}
$$

and

$$
\left(\partial_{n} \tilde{\boldsymbol{u}}^{n+1}, \tilde{\boldsymbol{u}}^{n+1}\right)_{\Gamma}=-\left\|l^{1 / 2}\left(\phi^{n}\right) \tilde{\boldsymbol{u}}_{s}^{n+1}\right\|_{\Gamma}^{2}+B\left(\tilde{L}^{n+1} \nabla_{\tau} \phi^{n}, \boldsymbol{u}_{\tau}^{n+1}\right)_{\Gamma} .
$$

(v) Next, we rewrite equation (3.11) as

$$
\frac{R}{\delta t} \boldsymbol{u}^{n+1}+\nabla p^{n+1}=\frac{R}{\delta t} \tilde{\boldsymbol{u}}^{n+1}+\nabla p^{n} .
$$

By integrating the square of the both sides of the above equation, and using conditions (3.12) and (3.13), we get

$$
\left\|\frac{R}{\delta t} \boldsymbol{u}^{n+1}\right\|^{2}+\left\|\nabla p^{n+1}\right\|^{2}=\left\|\frac{R}{\delta t} \tilde{\boldsymbol{u}}^{n+1}\right\|^{2}+\left\|\nabla p^{n}\right\|^{2}+\frac{2 R}{\delta t}\left(\tilde{\boldsymbol{u}}^{n+1}, \nabla p^{n}\right),
$$

i.e.

$$
\frac{R}{2 \delta t}\left(\left\|\boldsymbol{u}^{n+1}\right\|^{2}-\left\|\tilde{\boldsymbol{u}}^{n+1}\right\|^{2}\right)+\frac{\delta t}{2 R}\left(\left\|\nabla p^{n+1}\right\|^{2}-\left\|\nabla p^{n}\right\|^{2}\right)=\left(\nabla p^{n}, \tilde{\boldsymbol{u}}^{n+1}\right) .
$$


(vi) Summing up equations (3.16), (3.17), (3.22), (3.23), (3.24) and (3.25), we get

$$
\begin{aligned}
& \frac{R}{2 \delta t}\left(\left\|\boldsymbol{u}^{n+1}\right\|^{2}-\left\|\boldsymbol{u}^{n}\right\|^{2}+\left\|\tilde{\boldsymbol{u}}^{n+1}-\boldsymbol{u}^{n}\right\|^{2}\right)+\frac{B \varepsilon}{2 \delta t}\left(\left\|\nabla \phi^{n+1}\right\|^{2}-\|\nabla \phi\|^{2}+\left\|\nabla\left(\phi^{n+1}-\phi^{n}\right)\right\|^{2}\right) \\
& +\frac{B}{\delta t}\left(\hat{F}\left(\phi^{n+1}\right)-\hat{F}\left(\phi^{n}\right), 1\right)+\frac{B}{\delta t}\left(g\left(\phi^{n+1}\right)-g\left(\phi^{n}\right), 1\right)_{\Gamma}+\frac{\delta t}{2 R}\left(\left\|\nabla p^{n+1}\right\|^{2}-\left\|\nabla p^{n}\right\|^{2}\right) \\
=- & \left\|\nabla \tilde{\boldsymbol{u}}^{n+1}\right\|^{2}-M B\left\|\nabla \mu^{n+1}\right\|^{2}-B \gamma\left\|\tilde{L}^{n+1}\right\|_{\Gamma}^{2}-\left\|l^{1 / 2}\left(\phi^{n}\right) \tilde{\boldsymbol{u}}_{s}^{n+1}\right\|_{\Gamma}^{2} \\
& -B\left(s_{1}-\frac{\hat{f}^{\prime}(\xi)}{2}, \frac{\left(\phi^{n+1}-\phi^{n}\right)^{2}}{\delta t}\right)-B\left(s_{2}-\frac{g^{\prime \prime}(\zeta)}{2}, \frac{\left(\phi^{n+1}-\phi^{n}\right)^{2}}{\delta t}\right)_{\Gamma},
\end{aligned}
$$

which implies that

$$
\begin{aligned}
E_{t o t}^{n+1}-E_{t o t}^{n} & \\
=-\delta t & {\left[\left\|\nabla \tilde{\boldsymbol{u}}^{n+1}\right\|^{2}+M B\left\|\nabla \mu^{n+1}\right\|^{2}+B \gamma\left\|\tilde{L}^{n+1}\right\|_{\Gamma}^{2}+\left\|l^{1 / 2}\left(\phi^{n}\right) \tilde{\boldsymbol{u}}_{s}^{n+1}\right\|_{\Gamma}^{2}\right] } \\
- & \frac{R}{2} \delta t^{2}\left\|\frac{\tilde{\boldsymbol{u}}^{n+1}-\boldsymbol{u}^{n}}{\delta t}\right\|^{2}-\frac{B \varepsilon}{2} \delta t^{2}\left\|\frac{\nabla\left(\phi^{n+1}-\phi^{n}\right)}{\delta t}\right\|^{2} \\
& \quad-B \delta t^{2}\left(s_{1}-\frac{\hat{f}^{\prime}(\xi)}{2},\left(\frac{\phi^{n+1}-\phi^{n}}{\delta t}\right)^{2}\right)-B \delta t^{2}\left(s_{2}-\frac{g^{\prime \prime}(\xi)}{2},\left(\frac{\phi^{n+1}-\phi^{n}}{\delta t}\right)^{2}\right)_{\Gamma} .
\end{aligned}
$$

By the assumption $s_{1} \geq L_{1} / 2$ and $s_{2} \geq L_{2} / 2$, we get the desired energy estimate.

Note that there are three types of dissipations in equation (3.26). The first one is the $\mathrm{real} /$ physical dissipation which is consistent with (2.13). The second part is the dissipation due to implicit Euler discretization for $\boldsymbol{u}$ and $\phi$ equation. The third part is due to the artificial stabilization. Note that the second and third part are one order (in $\delta t$ ) smaller than the physical dissipation.

\subsection{A linear and decoupled energy stable scheme}

The LC scheme constructed above requires solving, at every time step, a coupled system for $\tilde{\boldsymbol{u}}^{n+1}, \phi^{n+1}, \mu^{n+1}$ with variable coefficients. In this subsection, we construct a linear and decoupled (LD) scheme, and prove that it is energy stable for systems with static contact line condition (2.11). For the system (2.1)-(2.8), our LD scheme calculates $\boldsymbol{u}^{n+1}, \phi^{n+1}, p^{n+1}$ and $\mu^{n+1}$ from $\boldsymbol{u}^{n}, \phi^{n}, p^{n}$ in three steps.

(i) We first solve for $\phi^{n+1}$ and $\mu^{n+1}$ from

$$
\begin{gathered}
\mu^{n+1}=-\varepsilon \Delta \phi^{n+1}+\hat{f}\left(\phi^{n}\right)+s_{1}\left(\phi^{n+1}-\phi^{n}\right), \\
\frac{\phi^{n+1}-\phi^{n}}{\delta t}+\nabla \cdot\left(\boldsymbol{u}_{*}^{n} \phi^{n}\right)=M \Delta \mu^{n+1},
\end{gathered}
$$

with boundary conditions

$$
\begin{gathered}
\partial_{n} \mu^{n+1}=0 \quad \text { on } \Gamma, \\
\frac{\phi^{n+1}-\phi^{n}}{\delta t}+\boldsymbol{u}_{\tau}^{n} \cdot \nabla_{\tau} \phi^{n}=-\gamma \tilde{L}^{n+1} \quad \text { on } \Gamma,
\end{gathered}
$$


where

$$
\boldsymbol{u}_{*}^{n}=\boldsymbol{u}^{n}-\delta t \frac{B}{R} \phi^{n} \nabla \mu^{n+1}
$$

(ii) Then, we solve $\tilde{\boldsymbol{u}}^{n+1}$ from

$$
R\left(\frac{\tilde{\boldsymbol{u}}^{n+1}-\boldsymbol{u}_{*}^{n}}{\delta t}+\left(\boldsymbol{u}^{n} \cdot \nabla\right) \tilde{\boldsymbol{u}}^{n+1}\right)-\Delta \tilde{\boldsymbol{u}}^{n+1}+\nabla p^{n}=0
$$

with boundary conditions

$$
\begin{gathered}
\tilde{\boldsymbol{u}}^{n+1} \cdot \boldsymbol{n}=0 \quad \text { on } \Gamma, \\
\partial_{n} \tilde{\boldsymbol{u}}_{\tau}^{n+1}+l\left(\phi^{n}\right) \tilde{\boldsymbol{u}}_{s}^{n+1}-B \tilde{L}^{n+1} \nabla_{\tau} \phi^{n}=0 \quad \text { on } \Gamma .
\end{gathered}
$$

(iii) In the last step, we update $\boldsymbol{u}^{n+1}$ and $p^{n+1}$ from (3.11)-(3.13), all the same as in the second step of the LC scheme.

The above LD scheme is derived from the LC scheme by replacing $\tilde{\boldsymbol{u}}^{n+1}$ with $\boldsymbol{u}_{*}^{n}$ in equation (3.5), and replacing $\tilde{\boldsymbol{u}}_{\tau}^{n+1}$ with $\boldsymbol{u}_{\tau}^{n}$ in equation (3.9). The introduction of an explicit velocity $\boldsymbol{u}_{*}^{n}$ in (3.28) follows a similar approach used in Minjeaud (2013) (see also Shen and Yang (2014a)). Comparing to the replacement of $\tilde{\boldsymbol{u}}^{n+1}$ with $\boldsymbol{u}^{n}$, the replacement of $\tilde{\boldsymbol{u}}^{n+1}$ with $\boldsymbol{u}_{*}^{n}$ in equation (3.5) adds an extra dissipation term $\left.\delta t B / R \nabla \dot{(}\left(\phi^{n}\right)^{2} \nabla \mu\right)$ to the equation. This allows us to prove a discrete energy dissipation law for systems with static contact line conditions. However, it remains open about how to extend this skill to handle $\tilde{\boldsymbol{u}}_{\tau}^{n+1}$ in equation (3.9). Thus direct explicit treatment is used. The numerical results in Section 5 show that the above LD scheme has very good stability property.

When $\gamma \rightarrow+\infty$, the above LD scheme is reduced to a scheme for the system consisting of (2.1)-(2.3), (2.5)-(2.7) with NBC (2.12) and static contact line condition (2.11) naturally. For this case, we have a guaranteed energy dissipation.

Theorem 3. Assume $s_{1} \geq L_{1} / 2, s_{2} \geq L_{2} / 2$ and $\boldsymbol{u}_{w}=\mathbf{0}$. Then, the scheme (3.27)-(3.34), (3.11)-(3.13) is energy stable for the case $\gamma \rightarrow+\infty$.

Proof. (i) Equation (3.27) is the same as equation (3.4), so the estimate (3.22) holds.

(ii) Taking the inner product of equation (3.28) with $B \mu^{n+1}$, noticing $\boldsymbol{u}_{*}^{n} \cdot \boldsymbol{n}=0$ on boundary and using (3.29), we have

$$
B\left(\frac{\phi^{n+1}-\phi^{n}}{\delta t}, \mu^{n+1}\right)-B\left(\boldsymbol{u}_{*}^{n} \phi^{n}, \nabla \mu^{n+1}\right)=-B M\left\|\nabla \mu^{n+1}\right\|^{2} .
$$

(iii) Taking the inner product of equation (3.32) with $\tilde{\boldsymbol{u}}^{n+1}$, we obtain

$$
\begin{aligned}
\frac{R}{2 \delta t}\left(\left\|\tilde{\boldsymbol{u}}^{n+1}\right\|^{2}-\left\|\boldsymbol{u}_{*}^{n}\right\|^{2}+\left\|\tilde{\boldsymbol{u}}^{n+1}-\boldsymbol{u}_{*}^{n}\right\|^{2}\right)=- & \left\|\nabla \tilde{\boldsymbol{u}}^{n+1}\right\|^{2}+\left(\partial_{n} \tilde{\boldsymbol{u}}^{n+1}, \tilde{\boldsymbol{u}}^{n+1}\right)_{\Gamma} \\
& -\left(\nabla p^{n}, \tilde{\boldsymbol{u}}^{n+1}\right) .
\end{aligned}
$$


Taking the inner product of equation (3.31) with $R \boldsymbol{u}_{*}^{n} / \delta t$, we have

$$
\frac{R}{2 \delta t}\left(\left\|\boldsymbol{u}_{*}^{n}\right\|^{2}-\left\|\boldsymbol{u}^{n}\right\|^{2}+\left\|\boldsymbol{u}_{*}^{n}-\boldsymbol{u}^{n}\right\|^{2}\right)=-B\left(\phi^{n} \nabla \mu^{n+1}, \boldsymbol{u}_{*}^{n}\right)
$$

(iv) The treatment of pressure term is same as in the LC scheme, so (3.25) still holds.

(v) For the boundary term in equation (3.36), equation (3.24) still holds. For the last boundary term in equation (3.22), using equation (3.30), we have the estimate

$$
\begin{aligned}
B\left(\tilde{L}^{n+1}, \frac{\phi^{n+1}-\phi^{n}}{\delta t}\right)_{\Gamma} & =B\left(\tilde{L}^{n+1},-\gamma \tilde{L}^{n+1}-\boldsymbol{u}_{\tau}^{n} \cdot \nabla_{\tau} \phi^{n}\right)_{\Gamma} \\
& =-B \gamma\left\|\tilde{L}^{n+1}\right\|_{\Gamma}^{2}-B\left(\tilde{L}^{n+1} \nabla_{\tau} \phi^{n}, \boldsymbol{u}_{\tau}^{n}\right)_{\Gamma} .
\end{aligned}
$$

(vi) Summing up equations (3.22), (3.25), (3.35), (3.36), (3.37) and (3.38), we get

$$
\begin{aligned}
E_{t o t}^{n+1}-E_{t o t}^{n} & \\
=- & \delta t\left[\left\|\nabla \tilde{\boldsymbol{u}}^{n+1}\right\|^{2}+M B\left\|\nabla \mu^{n+1}\right\|^{2}+\left\|l^{1 / 2}\left(\phi^{n}\right) \tilde{\boldsymbol{u}}_{s}^{n+1}\right\|_{\Gamma}^{2}\right] \\
& -\frac{R}{2} \delta t^{2}\left\|\frac{\tilde{\boldsymbol{u}}^{n+1}-\boldsymbol{u}_{*}^{n}}{\delta t}\right\|^{2}-\frac{R}{2} \delta t^{2}\left\|\frac{\boldsymbol{u}_{*}^{n}-\boldsymbol{u}^{n}}{\delta t}\right\|^{2}-\frac{B \varepsilon}{2} \delta t^{2}\left\|\frac{\nabla\left(\phi^{n+1}-\phi^{n}\right)}{\delta t}\right\|^{2} \\
& -B \delta t^{2}\left(s_{1}-\frac{\hat{f}^{\prime}(\xi)}{2},\left(\frac{\phi^{n+1}-\phi^{n}}{\delta t}\right)^{2}\right)-B \delta t^{2}\left(s_{2}-\frac{g^{\prime \prime}(\xi)}{2},\left(\frac{\phi^{n+1}-\phi^{n}}{\delta t}\right)^{2}\right)_{\Gamma} \\
& -B \gamma \delta t\left\|\tilde{L}^{n+1}\right\|_{\Gamma}^{2}-B \delta t^{2}\left(\tilde{L}^{n+1} \nabla_{\tau} \phi^{n}, \frac{\boldsymbol{u}_{\tau}^{n}-\tilde{\boldsymbol{u}}_{\tau}^{n+1}}{\delta t}\right)_{\Gamma} .
\end{aligned}
$$

The last term is a splitting error that can not be bounded by the other boundary dissipation terms in equation (3.39) for arbitrary time step $\delta t$ and small $\gamma$. But when $\gamma \rightarrow+\infty$, we have $\tilde{L}^{n+1}=0$ from (3.30), thus the last term disappears. Then by the assumption $s_{1} \geq L_{1} / 2$ and $s_{2} \geq L_{2} / 2$, we get the desired energy estimate.

Remark 2. When $\gamma$ is finite, we don't have $\tilde{L}^{n+1}=0$. To control the last term in equation (3.39), we must have a good estimate about $\nabla_{\tau} \phi^{n}\left(\boldsymbol{u}_{\tau}^{n}-\tilde{\boldsymbol{u}}_{\tau}^{n+1}\right)$ on $\Gamma$, which is not trivial. In fact, the numerical results in Section 5 show that the LD scheme is not unconditional stable for any $\delta t$ if $\gamma$ is not large enough (see Table 3).

\section{Spatial discretization}

Since the proofs of energy stability for our schemes are based on weak form and integration by parts, it can be speculated that a suitable spatial discretization based on Galerkin approximation of the NSCH coupled system will be able to keep the energy dissipation properties of the semi-discretized LC and LD schemes, although such a proof will be by no means trivial, especially when aliasing error exists.

In this section, we consider $\Omega=\left[0, L_{x}\right] \times[-1,1]$ with the periodic boundary condition in $x$, and provide some details for the implementation of a Fourier-Legendre Galerkin method 
for the spatial discretization to test the approximation properties of our time discretization schemes. We take the LD scheme (3.27)-(3.34), (3.11)-(3.13) as an example to describe the Fourier-Legendre Galerkin approximation. The construction of spectral Galerkin approximation for the LC scheme (3.3)-(3.13) is similar.

\subsection{Weak formulation}

We first rewrite the LD scheme in the weak form.

In the first step, we need to solve (3.27)-(3.30), which are equivalent to

$$
\begin{gathered}
-\varepsilon \Delta \phi^{n+1}+s_{1} \phi^{n+1}-\mu^{n+1}=s_{1} \phi^{n}-\hat{f}\left(\phi^{n}\right), \\
\frac{1}{\delta t} \phi^{n+1}-\delta t \frac{B}{R} \nabla \cdot\left(\beta\left(\phi^{n}\right) \nabla \mu^{n+1}\right)-M \Delta \mu^{n+1}=\frac{1}{\delta t} \phi^{n}-\nabla \cdot\left(\boldsymbol{u}^{n} \phi^{n}\right), \\
\partial_{n} \mu^{n+1}=0 \quad \text { on } \Gamma, \\
\left(\frac{1}{\gamma \delta t}+s_{2}\right) \phi^{n+1}+\varepsilon \partial_{n} \phi^{n+1}=\frac{1}{\gamma}\left(\frac{1}{\delta t} \phi^{n}-\boldsymbol{u}_{\tau}^{n} \cdot \nabla_{\tau} \phi^{n}\right)-\left(g^{\prime}\left(\phi^{n}\right)-s_{2} \phi^{n}\right) \quad \text { on } \Gamma,
\end{gathered}
$$

where $\beta\left(\phi^{n}\right)=\left(\phi^{n}\right)^{2}$. The corresponding weak formulation for the above equations reads:

Find $\mu^{n+1}, \phi^{n+1} \in H^{1}(\Omega)$, such that for any $\omega, \varphi \in H^{1}(\Omega)$

$$
\begin{gathered}
\varepsilon\left(\nabla \phi^{n+1}, \nabla \varphi\right)+s_{1}\left(\phi^{n+1}, \varphi\right)+c\left(\phi^{n+1}, \varphi\right)_{\Gamma}-\left(\mu^{n+1}, \varphi\right)=\left(s_{1} \phi^{n}-\hat{f}\left(\phi^{n}\right), \varphi\right)+\left(\phi_{\Gamma}^{n}, \varphi\right)_{\Gamma} \\
\frac{1}{\delta t}\left(\phi^{n+1}, \omega\right)+\delta t \frac{B}{R}\left(\beta\left(\phi^{n}\right) \nabla \mu^{n+1}, \nabla \omega\right)+M\left(\nabla \mu^{n+1}, \nabla \omega\right)=\left(\phi_{r}^{n}, \omega\right) .
\end{gathered}
$$

Here $c=1 / \gamma \delta t+s_{2}, \phi_{r}^{n}=\phi^{n} / \delta t-\nabla \cdot\left(\boldsymbol{u}^{n} \phi^{n}\right)$, and $\phi_{\Gamma}^{n}=\left(\phi^{n} / \delta t-\boldsymbol{u}_{\tau}^{n} \cdot \nabla_{\tau} \phi^{n}\right) / \gamma-g^{\prime}\left(\phi^{n}\right)+s_{2} \phi^{n}$. Note that to handle systems with static contact line conditions, we only need to set $1 / \gamma=0$ in the above formulation.

In the second step, we solve equations (3.31)-(3.34). The corresponding weak formulation reads:

Find $\tilde{\boldsymbol{u}}^{n+1} \in V_{\boldsymbol{u}}:=H^{1}(\Omega) \times H_{0}^{1}(\Omega)$, such that for any $\boldsymbol{v} \in V_{\boldsymbol{u}}$

$$
R\left(\frac{\tilde{\boldsymbol{u}}^{n+1}}{\delta t}+\boldsymbol{u}^{n} \cdot \nabla \tilde{\boldsymbol{u}}^{n+1}, \boldsymbol{v}\right)+\left(\nabla \tilde{\boldsymbol{u}}^{n+1}, \nabla \boldsymbol{v}\right)+\left(l\left(\phi^{n}\right) \tilde{\boldsymbol{u}}_{s}^{n+1}, \boldsymbol{v}_{\tau}\right)_{\Gamma}=\left(\boldsymbol{u}_{r}^{n}, \boldsymbol{v}\right)+\left(\boldsymbol{u}_{\Gamma}^{n}, \boldsymbol{v}_{\tau}\right)_{\Gamma},
$$

where $\boldsymbol{u}_{r}^{n}=R \boldsymbol{u}_{*}^{n} / \delta t-\nabla p^{n}, \boldsymbol{u}_{\Gamma}^{n}=B \tilde{L}^{n+1} \nabla_{\tau} \phi^{n}$.

In the last step, we solve (3.11)-(3.13). The corresponding weak form reads:

Find $p \in H_{c}^{1}(\Omega):=\left\{p: p \in H^{1}(\Omega), \int_{\Omega} p \mathrm{~d} x=0\right\}$, such that for any $q \in H_{c}^{1}(\Omega)$

$$
\left(\nabla p^{n+1}, \nabla q\right)=\left(\nabla p^{n}, \nabla q\right)-\frac{R}{\delta t}\left(\nabla \cdot \tilde{\boldsymbol{u}}^{n+1}, q\right)
$$




\subsection{Fourier-Legendre Galerkin approximation}

Since the system is periodic in the $x$-direction, we use

$$
F_{m}:=\operatorname{span}\left\{E_{k}(x)=e^{2 \pi i k x / L_{x}},|k| \leq m\right\}, \quad P_{k}=\operatorname{span}\left\{\varphi_{j}(y): 0 \leq j \leq k\right\}
$$

as the basis set for the $x$-direction and $y$-direction correspondingly, where

$$
\varphi_{0}(y)=\frac{1+x}{2}, \quad \varphi_{1}(y)=\frac{1-x}{2}, \quad \varphi_{j}(y)=L_{j}(y)-L_{j-2}(y), \quad j=2,3, \ldots,
$$

and $L_{j}(y)$ is the Legendre polynomial of degree $j$. This is a direct extension of nearly orthogonal bases used by Shen (1994). For given $m, k$, we take $F_{m} \otimes P_{k}$ as the approximation space for $\mu^{n+1}$ and $\phi^{n+1}$. For the Navier-Stokes equation, the velocity in $x$-component satisfies the GNBC, which is a Robin type boundary condition, while the component in $y$-direction satisfies Dirichlet boundary condition. The Robin type boundary condition is treated naturally in the weak form, the Dirichlet boundary condition is imposed on the approximation space. i.e. the Galerkin approximation space for $V_{\boldsymbol{u}}$ is $V_{m, k}^{\boldsymbol{u}}:=\{(u, v) \mid u \in$ $\left.F_{m} \otimes P_{k}, v \in F_{m} \otimes P_{k}^{0}\right\}$, where $P_{k}^{0}=\operatorname{span}\left\{\varphi_{j}(y), j=2, . ., k\right\}$. The approximation space for pressure is $V_{m, k}^{p}=F_{m} \otimes P_{k} \backslash C:=\operatorname{span}\left\{E_{l}(x) \varphi_{j}(y):|l| \leq m, 0 \leq j \leq k, l^{2}+j^{2} \neq 0\right\}$.

\subsection{Preconditioning}

Using the spectral bases defined in (4.9), the constant-coefficient terms in equations (4.5)-(4.8) all lead to sparse matrices and time independent. But the variable-coefficient terms in equation (4.6) and (4.7) lead to time-dependent dense matrices. Explicitly building those time-dependent dense matrices are expensive (Note that, if one use low-order finite element methods, the corresponding matrices will be sparse but still time-dependent). So we do not explicitly build them, but use the preconditioned matrix-free BiCGSTAB method proposed by van der Vorst (1992). The system matrices for the left hand sides of following equations will be used as preconditioners of (4.5)-(4.6), and (4.7) correspondingly.

Find $\mu^{n+1}, \phi^{n+1} \in F_{m} \otimes P_{k}$, such that for any $\omega, \varphi \in F_{m} \otimes P_{k}$

$$
\begin{gathered}
\varepsilon\left(\nabla \phi^{n+1}, \nabla \varphi\right)+s_{1}\left(\phi^{n+1}, \varphi\right)+c\left(\phi^{n+1}, \varphi\right)_{\Gamma}-\left(\mu^{n+1}, \varphi\right)=r_{1}[\varphi], \\
\frac{1}{\delta t}\left(\phi^{n+1}, \omega\right)+\tilde{M}\left(\nabla \mu^{n+1}, \nabla \omega\right)=r_{2}[\omega] .
\end{gathered}
$$

Here $\tilde{M}=\delta t B / R+M$.

Find $\tilde{\boldsymbol{u}}^{n+1} \in V_{m, k}^{\boldsymbol{u}}$, such that for any $\boldsymbol{v} \in V_{m, k}^{\boldsymbol{u}}$

$$
\frac{R}{\delta t}\left(\tilde{\boldsymbol{u}}^{n+1}, \boldsymbol{v}\right)+\left(\nabla \tilde{\boldsymbol{u}}^{n+1}, \nabla \boldsymbol{v}\right)+\bar{l}\left(\tilde{\boldsymbol{u}}_{s}^{n+1}, \boldsymbol{v}_{\tau}\right)_{\Gamma}=r_{3}[\boldsymbol{v}]
$$

where $\bar{l}=\max _{\phi}|l(\phi)|$, and $r_{1}[\varphi], r_{2}[\omega], r_{3}[\boldsymbol{v}]$ are linear functionals that are not involved in the preconditioning procedure. 
Similar procedure is used for the LC scheme. More precisely, we use (4.11), (4.12) with $\tilde{M}=M$, together with following equation as a preconditioner to the coupled linear system for $\phi^{n+1}, \mu^{n+1}, \tilde{\boldsymbol{u}}^{n+1}$ in the LC scheme.

$$
\frac{R}{\delta t}\left(\tilde{\boldsymbol{u}}^{n+1}, \boldsymbol{v}\right)+\left(\nabla \tilde{\boldsymbol{u}}^{n+1}, \nabla \boldsymbol{v}\right)+\bar{l}\left(\tilde{\boldsymbol{u}}_{s}^{n+1}, \boldsymbol{v}_{\tau}\right)_{\Gamma}-B\left(\tilde{L}^{n+1} \nabla_{\tau} \phi^{n}, \boldsymbol{v}_{\tau}\right)_{\Gamma}+B\left(\phi^{n} \nabla \mu^{n+1}, \boldsymbol{v}\right)=r_{4}[\boldsymbol{v}]
$$

Numerical results in the next section indicate that the preconditioners we choose here are very efficient and nearly optimal in the sense that the numbers of BiCGSTAB iterations are almost independent of spatial resolutions.

Remark 3. The energy stability analysis discussed in last section applies to the full discretizations as long as the Galerkin (not pseudo-Galerkin) formulations are used. In the numerical implementation, we use double quadrature points to numerically integrate variablecoefficient and nonlinear terms for simplicity. Since those terms are all explicit in the matrix-free BiCGSTAB solver, it is easy to improve the accuracy of numerical integration adaptively.

\section{Numerical results}

In this section, we present some numerical results using the two schemes constructed above, with double quadrature points for integrating variable-coefficient and nonlinear terms. Numerical results show that the aliasing error of double rule is very small for the spatial resolutions used in this section, and has no significant effect on the stability of the proposed schemes.

We consider the flow between two parallel plates which move in opposite directions at a constant speed. We fix the domain size to be $L_{y}=2, L_{x}=10$, and set default values of other dimensionless parameters as:

$$
B=12, M=0.0125, \gamma=100, l(\phi) \equiv 1 / 0.19, \quad R=0.6, \varepsilon=0.05 .
$$

This is similar to the configuration used in (He et al., 2011) and (Gao and Wang, 2012) after a dimensional rescaling. Particularly, This set of parameters corresponds to $L_{z}=0.4$, $L_{x}=2, \mathcal{B}=12, \mathcal{L}_{d}=5 \times 10^{-4}, \mathcal{V}_{s}=500, l_{s}=0.038, R=3, \varepsilon=0.01$ in Gao and Wang (2012).

We first present numerical results for two cases from Qian et al. (2006), He et al. (2011) and Gao and Wang (2012). In case $1, u_{w}= \pm 0.7, \theta_{s}=64^{\circ}$, where $u_{w}$ is the speed of top and bottom plates, $\theta_{s}$ is the static contact angle ; \pm sign means the values on top boundary and bottom boundary have different signs (directions). In case $2, u_{w}= \pm 0.2$ and $\theta_{s}= \pm 77.6^{\circ}$. In both case, the initial velocity field takes the profile of Couette flow, and the initial value of $\phi$ is given by (as shown in the top contour of Figure 1)

$$
\phi_{0}(x, y)=\tanh \left(\frac{1}{\sqrt{2} \varepsilon}\left(0.25 L_{x}-\left|x-0.5 L_{x}\right|\right)\right) .
$$


The contours of phase variable $\phi$ at $t=5$ obtained by the LC scheme with 257 Fourier modes $(m=128)$ and 32 Legendre modes with $\delta t=0.01$ for both cases are presented in Figure 1. The LD scheme gives similar results, so we only show the results of the LC scheme. The results are consistent to the Figure 5 in Qian et al. (2006), Figure 2 in He et al. (2011) and Figure 2(a) in Gao and Wang (2012).

In Figure 2, we show the velocity and pressure at $t=10$ obtained from the LC scheme with 257 Fourier modes, 32 Legendre modes and $\delta t=0.02$ for the case $u_{w}= \pm 0.2, \theta_{s}=77.6^{\circ}$. Note that, the pressure in this paper is different to the pressure in existing literature (cf., for instance, Qian et al. (2003, 2006); He et al. (2011); Gao and Wang (2012), etc) by a value of $B \mu \phi$. The numerical results of the LD scheme are similar. Both schemes conserve the volume of $\phi$ to machine accuracy.
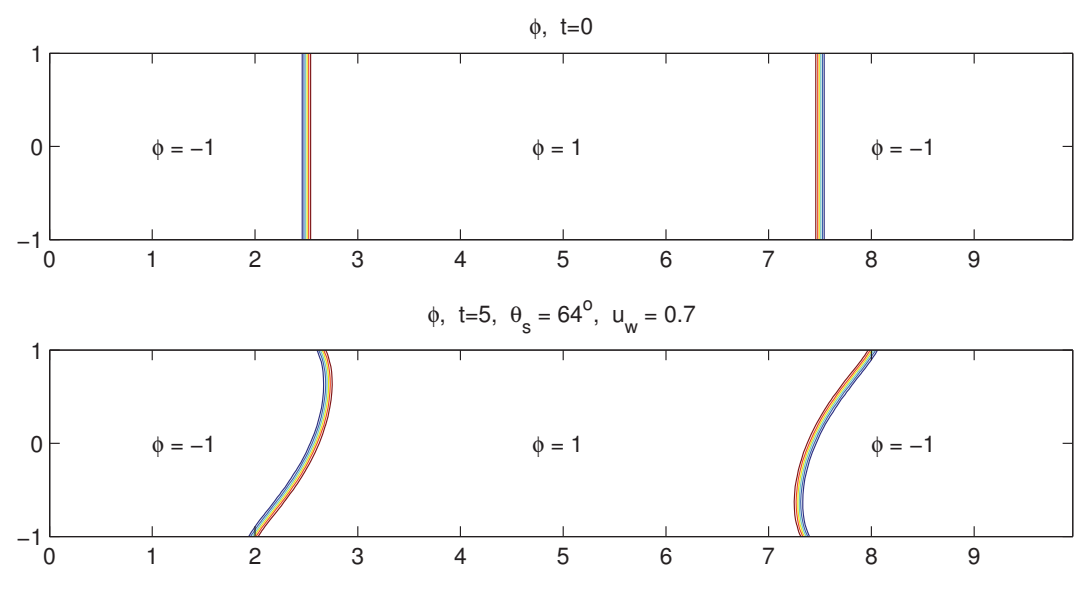

$\phi, t=5, \theta_{s}= \pm 77.6^{\circ}, u_{w}=0.2$

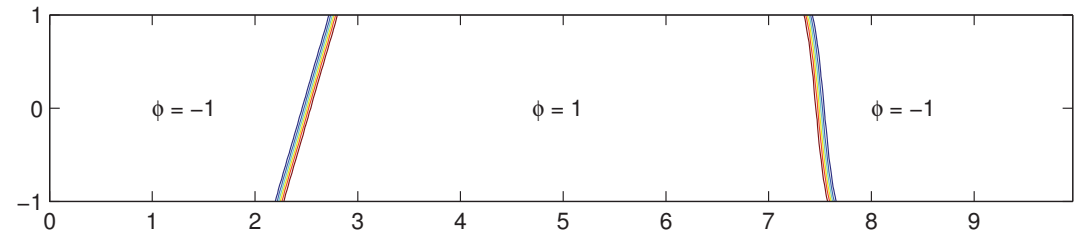

Figure 1: The contours of phase variable $\phi$ in two simulations. Top) The initial configuration of $\phi$ given by (5.2); Middle) Contour of $\phi$ at $t=5$ for the case: $\theta_{s}=64^{\circ}$ and $u_{w}= \pm 0.7$; Bottom) Contour of $\phi$ at $t=5$ for the case: $\theta_{s}(y= \pm 1)= \pm 77.6^{\circ}$ and $u_{w}= \pm 0.2$. The results are generated by the LC scheme with 257 Fourier modes and 32 Legendre modes with $\delta t=0.01$.

In Figure 3, we plot the $x$-component of velocity at lower boundary $y=-1$ for two different time steps $\delta t=0.02$ and $\delta t=0.01$ obtained from the LC scheme and LD scheme for the case $u_{w}= \pm 0.2, \theta_{s}=77.6^{\circ}$. We observe that the differences are very small but the convergence of the LC scheme appears to be slightly faster than the convergence of the LD scheme. The slower convergence of the LD scheme near the contact points is due to the extra dissipation introduced by $\boldsymbol{u}_{*}^{n}$. Particularly, in current parameter setting, the constant for the extra dissipation has a coefficient $\delta t B / R=20 \delta t$, which is larger than the other error introduced by the first order splitting. 

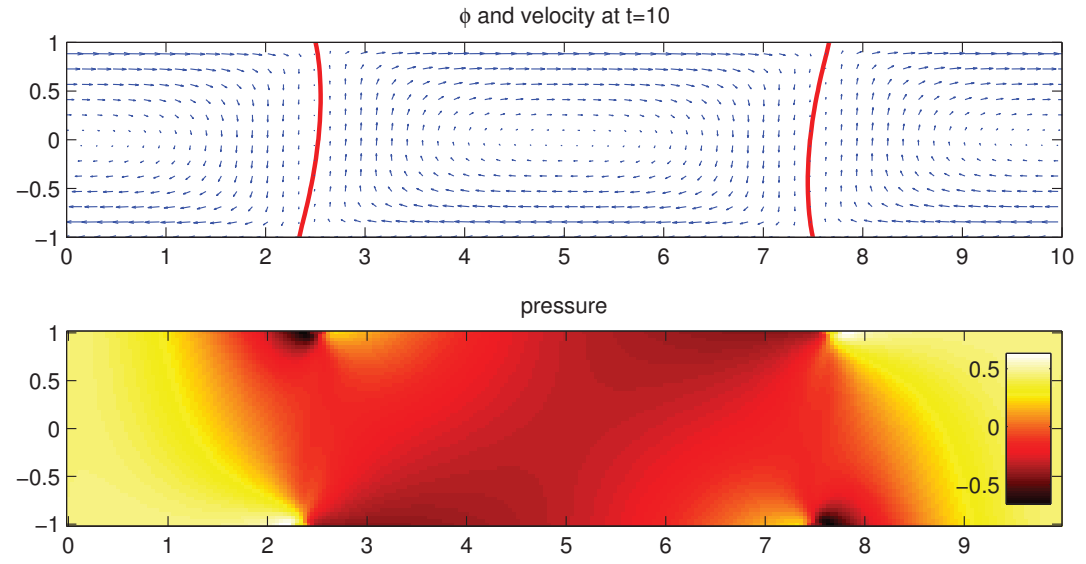

Figure 2: The information of $\phi, p, u, v$ fields at $t=10$ obtained from the LC scheme using 257 Fourier modes, 32 Legendre modes and time step size $\delta t=0.02$ for the case $\theta_{s}=77.6^{\circ}, u_{w}= \pm 0.2$. Other dimensionless parameters are given in (5.1). In the top picture, Two solid curves (red) are the interfaces of two phases (where $\phi=0$ ), arrows (blue) denote the directions and strength of the velocity at corresponding spatial points. In the bottom picture, color denotes the value of pressure. In both pictures, the horizontal axis is $x$, vertical axis is $y$.
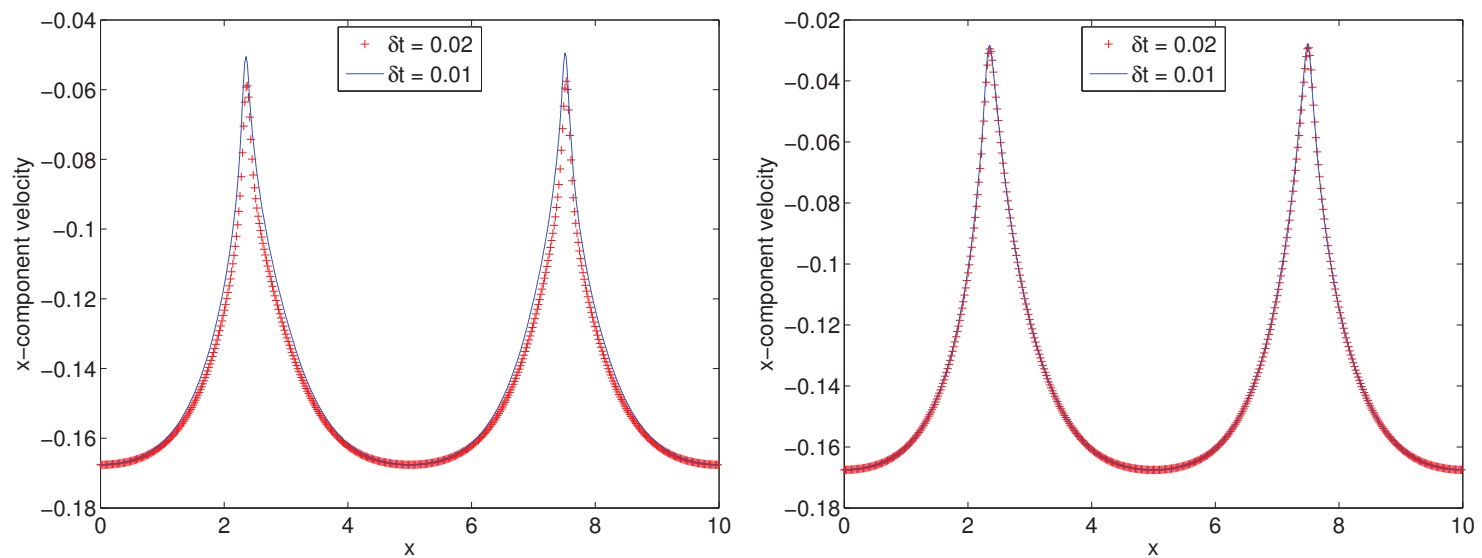

Figure 3: The $x$-component velocity at $y=-1, t=10$ using the LD scheme (left) and the LC scheme (right) with $\delta t=0.02$ and $\delta t=0.01$ for the case $\theta_{s}=77.6^{\circ}, u_{w}= \pm 0.2$. 
In Table 1, we show the temporal convergence property of the LC and LD schemes. We observe from this table that both schemes are first order accuracy in time. We note that the LD scheme is slightly worse than the LC scheme due to the error introduced by the extra stabilization term.

\begin{tabular}{c|cc|cc}
\hline$\delta t$ & $\left\|u_{L C}-u_{L C}^{e}\right\|_{2}$ & Order & $\left\|u_{L D}-u_{L D}^{e}\right\|_{2}$ & Order \\
\hline 0.008 & 0.0261 & 0.75 & 0.0185 & 0.50 \\
0.004 & 0.0155 & 1.15 & 0.0131 & 0.88 \\
0.002 & 0.0070 & 1.49 & 0.0071 & 1.29 \\
0.001 & 0.0025 & & 0.0029 & \\
\hline
\end{tabular}

Table 1: Numerical errors for velocity $u$ at $t=0.8$ for different time steps. The numerical results using $\delta t=0.0005$ are taken as reference solutions $u_{L C}^{e}, u_{L D}^{e}$. Spatial resolution $n_{x}=2 m+1=513, n_{y}=k+1=64$ are used for generating all the results.

In Table 2, we show the convergence of the spectral discretization. Only the results of the LC scheme are presented, since results for the LD scheme are similar. We observe from this table that, despite the near singular shape of the solution at contact points, our spectral method converges very fast, achieving good accuracy with a reasonably small number of unknowns. Note that with the phase field approach, the contact line moves naturally on the solid surface due to a diffusive flux across the interface driven by the gradient of the chemical potential (cf. Jacqmin (2000), Yue et al. (2010)), so there is no singularity with a fixed interfacial width.

\begin{tabular}{rcc|ccc}
\hline$n_{x} \times n_{y}$ & $\left\|u-u^{e}\right\|_{2}$ & $\left\|\phi-\phi^{e}\right\|_{2}$ & $n_{x} \times n_{y}$ & $\left\|u-u^{e}\right\|_{2}$ & $\left\|\phi-\phi^{e}\right\|_{2}$ \\
\hline $40 \times 64$ & $1.3 \mathrm{E}-1$ & $3.6 \mathrm{E}-1$ & $257 \times 8$ & $2.2 \mathrm{E}-3$ & $2.9 \mathrm{E}-3$ \\
$80 \times 64$ & $2.3 \mathrm{E}-2$ & $8.9 \mathrm{E}-2$ & $257 \times 16$ & $3.6 \mathrm{E}-4$ & $4.7 \mathrm{E}-4$ \\
$120 \times 64$ & $6.1 \mathrm{E}-3$ & $1.7 \mathrm{E}-2$ & $257 \times 24$ & $1.2 \mathrm{E}-4$ & $1.6 \mathrm{E}-4$ \\
$160 \times 64$ & $1.9 \mathrm{E}-4$ & $4.1 \mathrm{E}-3$ & $257 \times 32$ & $4.9 \mathrm{E}-5$ & $6.5 \mathrm{E}-4$ \\
\hline
\end{tabular}

Table 2: Convergence of spatial discretization. The results on fine grids $n_{x} \times n_{y}=240 \times 64$ and $n_{x} \times n_{y}=$ $257 \times 48$ are taken as the reference solutions $u^{e}, \phi^{e}$ for the left and right parts in the table correspondingly. All the errors are calculated at $t=1$ using time step size $\delta t=0.0005$.

From Theorems 2 and 3, we know that the LC scheme is unconditionally stable for any value of $\gamma$, and the LD scheme is unconditionally stable for the case $\gamma \rightarrow+\infty$. In Table 3 , we show the maximum stable time steps for the LD scheme with different values of $\gamma, B$ and different spatial resolutions. From this table we see that the spatial resolution almost has no effect on the stability of the LD scheme, and larger maximum time steps are allowed for larger $\gamma$ and smaller $B$. In particular, when $\gamma$ is much larger than $B$, the LD scheme becomes unconditionally stable.

In Table 4, we show average numbers of the inner iterations of BiCGSTAB for the $\phi, \mu$ equations in the LD scheme and the $\boldsymbol{u}, \phi, \mu$ equations in the LC scheme. We observe that 


\begin{tabular}{c|c|c|c|c|c|c|c|c|c}
\hline$n_{x} \times n_{y}$ & \multicolumn{3}{|c|}{$129 \times 16$} & \multicolumn{3}{c|}{$257 \times 32$} & \multicolumn{3}{c}{$513 \times 64$} \\
\hline$\gamma$ & 1 & 10 & 100 & 1 & 10 & 100 & 1 & 10 & 100 \\
\hline$B=1$ & $2.8 \mathrm{E}-1$ & $>100$ & $>100$ & $2.3 \mathrm{E}-1$ & $>100$ & $>100$ & $2.3 \mathrm{E}-1$ & $>100$ & $>100$ \\
$B=12$ & $2.2 \mathrm{E}-2$ & $2.7 \mathrm{E}-2$ & $>100$ & $1.8 \mathrm{E}-2$ & $2.2 \mathrm{E}-2$ & $>100$ & $1.8 \mathrm{E}-2$ & $2.2 \mathrm{E}-2$ & $>100$ \\
$B=144$ & $2.5 \mathrm{E}-3$ & $2.5 \mathrm{E}-3$ & $3.2 \mathrm{E}-3$ & $2.0 \mathrm{E}-3$ & $2.1 \mathrm{E}-3$ & $2.6 \mathrm{E}-3$ & $2.0 \mathrm{E}-3$ & $2.0 \mathrm{E}-3$ & $2.5 \mathrm{E}-3$ \\
\hline
\end{tabular}

Table 3: Maximum time steps allowed for different values of $\gamma, B$ and different spatial resolutions in the LD scheme.

the iterative solvers for the two schemes have similar performance. They all weakly depend on values of $\delta t$ and $B$, but are almost independent of spatial resolutions and the values of $\gamma$. Note that the BiCGSTAB solvers for the two velocity equations in the LD scheme converge in just 1-2 iterations. Considering that the computational cost of one inner iteration in LC scheme is almost three times the computational cost of one inner iteration for $\phi, \mu$ solver in the LD schemes, so the overall computational cost of the LD scheme is about $1 / 3$ the cost of the LC scheme.

\begin{tabular}{c|ccc|c|ccc}
\hline$n_{x} \times n_{y}$ & $129 \times 16$ & $257 \times 32$ & $513 \times 64$ & $\gamma$ & 100 & 10 & 1 \\
\hline $\mathrm{LD}$ & 5 & 7 & 9.5 & $\mathrm{LD}$ & 7 & 7 & 6.5 \\
$\mathrm{LC}$ & 5 & 5.5 & 6 & $\mathrm{LC}$ & 6 & 6.5 & 6.5 \\
\hline$\delta t$ & 0.001 & 0.1 & 10 & $B$ & 1 & 12 & 144 \\
\hline $\mathrm{LD}$ & 3.5 & 12.5 & 18 & $\mathrm{LD}$ & 3.5 & 7 & 9 \\
$\mathrm{LC}$ & 2.5 & 18 & 82 & $\mathrm{LC}$ & 2.5 & 5.5 & 18 \\
\hline
\end{tabular}

Table 4: Average numbers of inner iterations for BiCGSTAB solvers to converge to $10^{-9}$ in the LC and LD schemes. Except explicitly specified in the table, the values of $n_{x}, n_{y}, \delta t, \gamma, B$ are $n_{x}=257, n_{y}=32$, $\delta t=0.01, \gamma=500$ and $B=12$. For the LD scheme, only the iteration numbers for the Cahn-Hilliard equation are presented. The iteration numbers for the velocity equation are not presented, since they are almost unchanged (about 1-2 inner iterations) in these simulations.

Before ending this section, we present some numerical results of the dewetting and spreading process in Figure 4. On the left part, seven snapshots show the dewetting process of a droplet with static contact angle $\theta_{s}=30^{\circ}$; On the right part, seven snapshots show us the spreading process of a droplet with static contact angle $\theta_{s}=150^{\circ}$.

\section{Concluding remarks}

In this paper, we constructed two efficient temporal discretization schemes for a phasefield model incorporating MCLs. The model couples incompressible Navier-Stokes equations with GNBC and Cahn-Hilliard equation with static/dynamic contact line boundary conditions. The LC scheme and LD scheme are shown to obey a discrete energy dissipation law, thus allow for large time steps. The LC scheme is found to be slightly more robust and 
$\phi, \quad t=0$
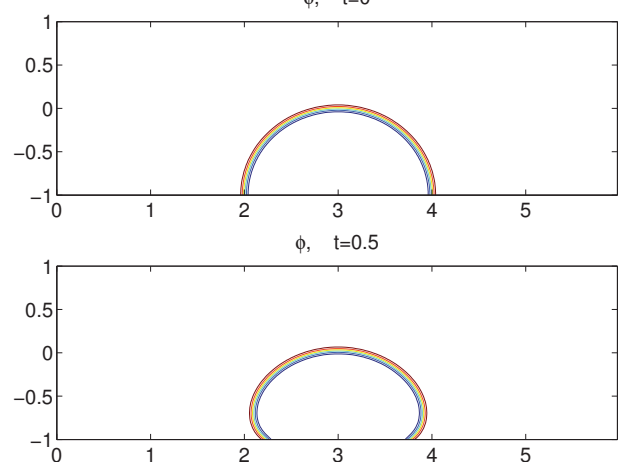

$\phi, \quad t=1$

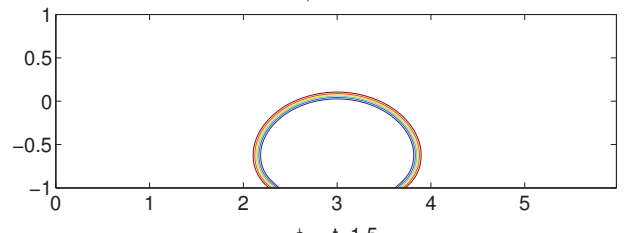

$\phi, \quad t=1.5$

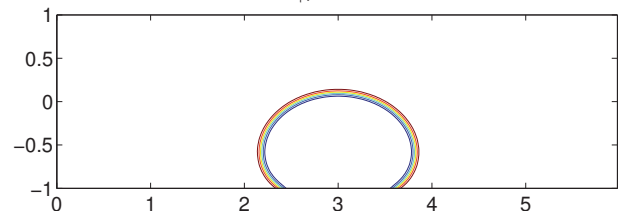

$\phi, \quad t=2$

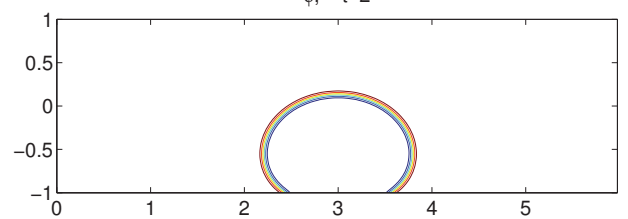

$\phi, \quad t=2.5$

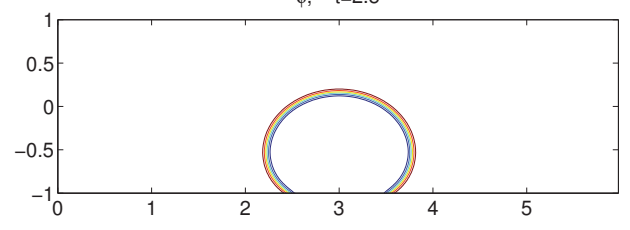

$\phi, \quad t=5$

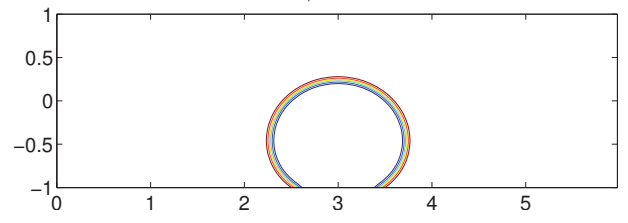

$\phi, \quad t=0$

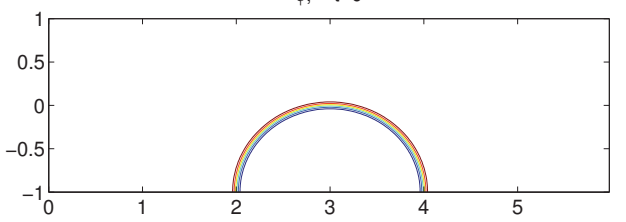

$\phi, \quad t=0.5$

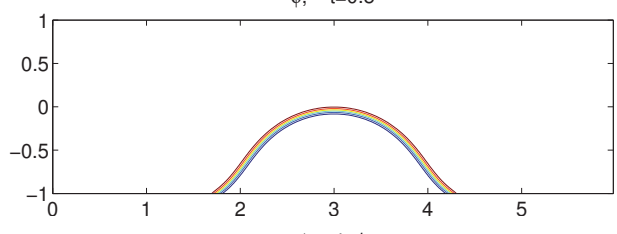

$\phi, \quad t=1$

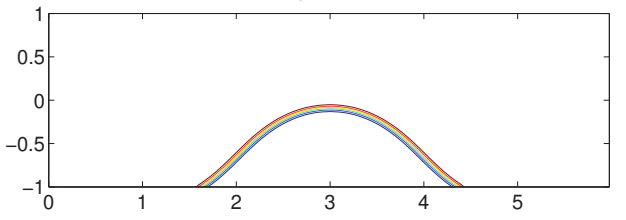

$\phi, \quad \mathrm{t}=1.5$

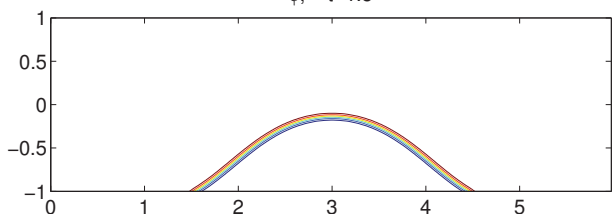

$\phi, \quad t=2$

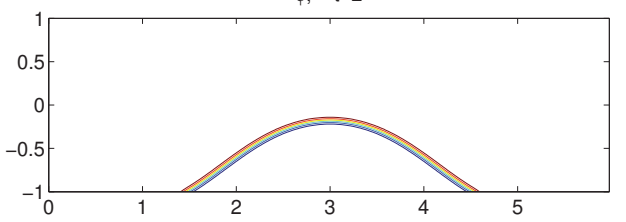

$\phi, \quad t=2.5$

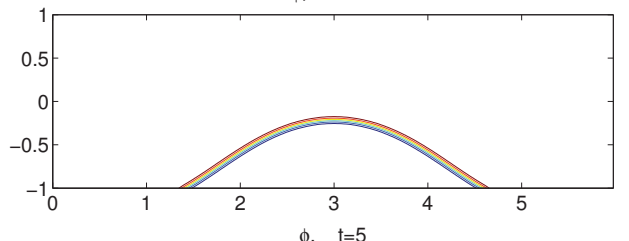

$\phi, \quad t=5$

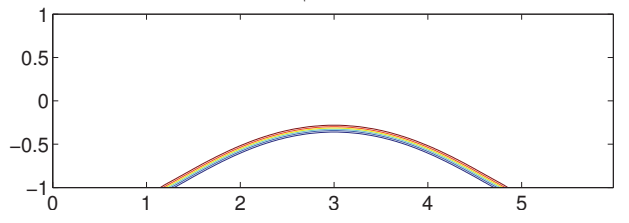

Figure 4: Spreading and dewetting of a droplet. Left) $\theta_{s}=30^{\circ}$; Right) $\theta_{s}=150^{\circ}$. We set $u_{w}=0$ in both cases. The other model parameters are defined in (5.1). The computational domain is $[0,6] \times[-1,1]$ as shown in the figures. The LC scheme with resolution $n_{x}=257, n_{y}=128, \delta=0.01$ is used to generate these results. 
accurate than the LD scheme, but it needs a relatively larger computational cost, while the LD scheme is more computational efficient but less robust for small $\gamma$ cases (when dynamical effect of contact line is significant) and relatively less accurate for the large $B / R$ case (large capillary force case).

Comparing to other methods using convex splitting (cf. He et al. (2011), Gao and Wang (2012), etc), the two energy stable schemes proposed in this paper only need to solve linear systems in each time step. Using the Fourier-Legendre Galerkin method as an example, we show that these linear systems can be solved efficiently by using a preconditioned BiCGSTAB solver. Since the schemes are discretized in a conserved form for the phase field variable, the volume fraction is conserved up to machine accuracy. We also verified that the spectral Galerkin approximation in space has very good convergence property despite the large gradient of $x$-component velocity near the contact points on boundaries.

The 2-dimensional numerical experiments in this paper show that the LC scheme and the LD scheme have similar efficiency-accuracy balance. But we expect the LD scheme to have better accuracy-efficiency balance for problems in complex 3-dimensional domains.

\section{Acknowledgments}

The work of J. Shen is supported in part by NSF DMS-1217066 and DMS-1419053. The work of X. Yang is partially supported by NSF DMS-1200487, NSF DMS-1418898, AFOSR FA9550-12-1-0178 and SC Epscor Gear fund. The work of $\mathrm{H}$. Yu is partially supported by the NSF of China under Grants 11101413 and 11371358.

\section{References}

Aland, S., Feng, C., 2014. An efficient and energy stable scheme for a phase-field model for the moving contact line problem. preprint.

Bray, A., 1994. Theory of phase-ordering kinetics. Advances in Physics 43 (3), 357-459.

Caffarelli, L. A., Muler, N. E., Dec. 1995. An $L^{\infty}$ bound for solutions of the Cahn-Hilliard equation. Arch. Rational Mech. Anal. 133 (2), 129-144.

Cahn, J. W., Hilliard, J. E., Feb. 1958. Free energy of a nonuniform system. I. interfacial free energy. The Journal of Chemical Physics 28 (2), 258-267.

Condette, N., Melcher, C., Süli, E., 2011. Spectral approximation of pattern-forming nonlinear evolution equations with double-well potentials of quadratic growth. Math. Comp. 80 (273), 205-223.

Dong, S., Nov. 2012. On imposing dynamic contact-angle boundary conditions for wall-bounded liquid-gas flows. Computer Methods in Applied Mechanics and Engineering 247-248, 179-200.

Dong, S., Shen, J., 2012. A time-stepping scheme involving constant coefficient matrices for phase-field simulations of two-phase incompressible flows with large density ratios. Journal of Computational Physics 231 (17), 5788-5804.

Du, Q., Nicolaides, R. A., 1991. Numerical analysis of a continuum model of phase transition. SIAM Journal on Numerical Analysis 28 (5), 1310-1322.

Dussan, E. B., 1979. On the spreading of liquids on solid surfaces: Static and dynamic contact lines. Annual Review of Fluid Mechanics 11 (1), 371-400.

Dussan V., E. B., Davis, S. H., 1974. On the motion of a fluid-fluid interface along a solid surface. Journal of Fluid Mechanics 65 (01), 71-95.

Elliott, C. M., Stuart, A. M., 1993. The global dynamics of discrete semilinear parabolic equations. SIAM J. Numer. Anal. 30, 1622-1663. 
Eyre, D. J., 1998. Unconditionally gradient stable time marching the Cahn-Hilliard equation. MRS Online Proceedings Library 529.

Furihata, D., Feb. 2001. A stable and conservative finite difference scheme for the Cahn-Hilliard equation. Numer. Math. 87 (4), 675-699.

Gao, M., Wang, X.-P., Feb. 2012. A gradient stable scheme for a phase field model for the moving contact line problem. Journal of Computational Physics 231 (4), 1372-1386.

Gao, M., Wang, X.-P., 2014. An efficient scheme for a phase field model for the moving contact line problem with variable density and viscosity. Journal of Computational Physics 272, 704-718.

Guermond, J., Minev, P., Shen, J., Sep. 2006. An overview of projection methods for incompressible flows. Computer Methods in Applied Mechanics and Engineering 195 (44-47), 6011-6045.

Hadjiconstantinou, N. G., Sep. 1999. Hybrid atomistic-continuum formulations and the moving contact-line problem. Journal of Computational Physics 154 (2), 245-265.

He, Q., Glowinski, R., Wang, X.-P., Jun. 2011. A least-squares/finite element method for the numerical solution of the Navier-Stokes-Cahn-Hilliard system modeling the motion of the contact line. Journal of Computational Physics 230 (12), 4991-5009.

He, Y., Liu, Y., Tang, T., May 2007. On large time-stepping methods for the Cahn-Hilliard equation. Applied Numerical Mathematics 57 (5-7), 616-628.

Jacqmin, D., 2000. Contact-line dynamics of a diffuse fluid interface. Journal of Fluid Mechanics 402, 57-88.

Kessler, D., Nochetto, R. H., Schmidt, A., 2004. A posteriori error control for the Allen-Cahn problem: circumventing gronwall's inequality. ESAIM: Mathematical Modelling and Numerical Analysis 38 (01), 129-142.

Koplik, J., Banavar, J. R., Willemsen, J. F., Mar. 1988. Molecular dynamics of poiseuille flow and moving contact lines. Phys. Rev. Lett. 60 (13), 1282-1285.

Koplik, J., Banavar, J. R., Willemsen, J. F., May 1989. Molecular dynamics of fluid flow at solid surfaces. Physics of Fluids A: Fluid Dynamics (1989-1993) 1 (5), 781-794.

Liu, C., Shen, J., May 2003. A phase field model for the mixture of two incompressible fluids and its approximation by a Fourier-spectral method. Physica D: Nonlinear Phenomena 179 (3-4), 211-228.

Minjeaud, S., Mar. 2013. An unconditionally stable uncoupled scheme for a triphasic Cahn-Hilliard/NavierStokes model. Numer. Methods Partial Differential Eq. 29 (2), 584-618.

Moffatt, H. K., 1964. Viscous and resistive eddies near a sharp corner. Journal of Fluid Mechanics 18 (01), $1-18$.

Qian, T., Wang, X.-P., Sheng, P., 2003. Molecular scale contact line hydrodynamics of immiscible flows. Physical Review E 68 (1), 016306.

Qian, T., Wang, X.-P., Sheng, P., 2006. A variational approach to moving contact line hydrodynamics. Journal of Fluid Mechanics 564, 333-360.

Rayleigh, L., Feb. 1892. XX. on the theory of surface forces II. compressible fluids. Philosophical Magazine Series 533 (201), 209-220.

Ren, W., E, W., Mar. 2005. Heterogeneous multiscale method for the modeling of complex fluids and micro-fluidics. Journal of Computational Physics 204 (1), 1-26.

Salgado, A. J., May 2013. A diffuse interface fractional time-stepping technique for incompressible twophase flows with moving contact lines. ESAIM: Mathematical Modelling and Numerical Analysis 47 (3), $743-769$.

Shen, J., 1994. Efficient spectral-galerkin method I. direct solvers of second- and fourth-order equations using Legendre polynomials. SIAM Journal on Scientific Computing 15 (6), 1489.

Shen, J., 2011. Modeling and numerical approximation of two-phase incompressible flows by a phase-field approach. In: Multiscale Modeling and Analysis for Materials Simulation, Lecture Note Series, Vol. 9. IMS, National University of Singapore, Edited by W. Bao and Q. Du. pp. 147-196.

Shen, J., Yang, X., Sep. 2010a. Energy stable schemes for Cahn-Hilliard phase-field model of two-phase incompressible flows. Chin. Ann. Math. Ser. B 31 (5), 743-758.

Shen, J., Yang, X., 2010b. Numerical approximations of Allen-Cahn and Cahn-Hilliard equations. Discrete Continuous Dynamical. Syst. A 28, 1669-1691. 
Shen, J., Yang, X., 2010c. A phase-field model and its numerical approximation for two-phase incompressible flows with different densities and viscosities. SIAM Journal on Scientific Computing 32 (3), 1159-1179.

Shen, J., Yang, X., 2014a. Decoupled energy stable schemes for phase-field models of two-phase complex fluids. SIAM Journal on Scientific Computing 36 (1), B122-B145.

Shen, J., Yang, X., 2014b. Decoupled, energy stable schemes for phase-field models of two-phase incompressible flows. Submitted.

Thompson, P. A., Robbins, M. O., Aug. 1989. Simulations of contact-line motion: Slip and the dynamic contact angle. Phys. Rev. Lett. 63 (7), 766-769.

van der Vorst, H., 1992. Bi-CGSTAB: A fast and smoothly converging variant of bi-CG for the solution of nonsymmetric linear systems. SIAM J. Sci. and Stat. Comput. 13 (2), 631-644.

Waals, J. D. v. d., Feb. 1893. The thermodynamic theory of capillarity under the hypothesis of a continuous variation of density. Transl. by J. S. Rowlinson J Stat Phys 20 (2), 200-244.

Xu, C., Tang, T., 2006. Stability analysis of large time-stepping methods for epitaxial growth models. Liq. Cryst. 44, 1759-1779.

Yue, P., Zhou, C., Feng, J. J., 2010. Sharp-interface limit of the Cahn-Hilliard model for moving contact lines. Journal of Fluid Mechanics 645, 279-294.

Zhu, J., Chen, L.-Q., Shen, J., Tikare, V., Oct. 1999. Coarsening kinetics from a variable-mobility CahnHilliard equation: Application of a semi-implicit fourier spectral method. Phys. Rev. E 60 (4), 3564-3572. 